\section{¿En qué consiste la presión tópica negativa? ¿Es eficaz/ eficiente en el cierre de heridas complejas? Revisión del tema}

\author{
What is the Pressure Topical \\ Negative? Is it effective/efficient \\ in wound closure complex? \\ Topic Review
}

\author{
Carmen María Sarabia Cobo ${ }^{1}$ \\ Cristina Castanedo Pfeiffer ${ }^{2}$ \\ 1. Ayudante LOU. Área Enfermería del Envejecimiento. Departamento de Enfermería. \\ Universidad de Cantabria. \\ 2. Profesora titular. Área Enfermería del Envejecimiento. Departamento de Enfermería. \\ Universidad de Cantabria.
}

\section{Correspondencia:}

Carmen María Sarabia Cobo

EUE Casa de Salud Valdecilla

Avda. Valdecilla, s/n

39011 Santander (Cantabria)

E-mail: carmen.sarabia@unican.es

\section{RESUMEN}

El objetivo del presente artículo es realizar una revisión sobre la terapia con presión tópica negativa en el tratamiento de úlceras y heridas complejas y crónicas. La necesidad de dar respuestas eficaces por un lado y ser costo-efectivos por otro está dando lugar en la actualidad a un debate sobre lo que supone curar integralmente una herida crónica. En muchos países cobra relevancia la búsqueda de tratamientos que aunque de entrada sean más caros que la cura tradicional, a la larga reducen los costes y las complicaciones derivadas. Asimismo, se presentan los tratamientos más conocidos, su eficacia en la reducción del gasto, así como las marcas comercializadas en España.

PALABRAS CLAVE: terapia de vacío, tratamiento con presión negativa, heridas complejas, úlceras por presión, estudios de costeefectividad.

\section{ABSTRACT}

The purpose of this article is to review the topical negative pressure therapy in the treatment of chronic and complex wounds. The need for effective responses on the one hand and to be cost effective on the other is leading to a debate about what is currently fully heal a chronic wound. In many countries becomes relevant finding that although input treatments are more expensive than traditional cure, ultimately reducing costs and complications. It also presents the most popular treatments, their effectiveness in reducing spending and brands marketed in Spain.

KEYWORDS: Vacuum assisted therapy, negative pressure therapy, complex wounds, pressure ulcers, cost effectiveness studies.

\section{У INTRODUCCIÓN}

Según un estudio realizado en EE.UU., solamente las heridas crónicas afectan a un $0,78 \%$ de la población americana (más de 265000 personas). En las personas mayores, las heridas crónicas suelen aparecer de manera secundaria a úlceras por presión (UPP) y lesiones de origen vascular.

El impacto de este tipo de lesiones de larga duración y abordaje complejo es elevado desde el punto de vista de calidad de vida, complicaciones secundarias y gasto sanitario. Por ejemplo, el costo del tratamiento de las UPP en España es de aproximadamente 461 millones de euros al año (1), y en el Reino Unido (2), el costo del cuidado de heridas es de más de mil millones de libras esterlinas por ańo, por lo que se requieren tratamientos clínicamente efectivos y poco costosos. El manejo de pacientes con heridas crónicas y dehiscencias quirúrgicas puede, en ocasiones, ser frustrante y caro.

La curación de las heridas va a depender en gran medida de las condiciones del lecho, de la microcirculación y del aporte vascular, y de la presencia de infección y carga bacteriana.
Actualmente existen tres tipos de abordaje de las heridas:

1. La cura tradicional o seca que hace uso de apósitos o gasas de una manera pasiva sólo cubre la herida y no tiene interacción con ésta. La cura suele ser diaria.

2. La cura avanzada, o en medio húmedo, es aquella que se realiza con apósitos o gasas humedecidas en suero fisiológico que tratan de mantener un ambiente húmedo en la herida. A este tipo de apósitos se les denomina activos porque interactúan con la lesión para favorecer la cicatrización. Entre sus propiedades destacan que son estériles, tienen capacidad de absorción, pueden proteger contra la infección o manejar la carga bacteriana, no son tóxicos ni alergénicos, disminuyen el dolor y el olor, y son fáciles de usar. No suelen emplear sustancias tópicas y no necesariamente requieren cambio diario.

3. Las técnicas avanzadas en cura de heridas exigen una tecnología avanzada. Entre ellas tenemos la terapia con oxígeno hiperbárico y la curación de heridas con presión tópica negativa (PTN).

El objetivo de este artículo es revisar la aplicación, uso, indicación y coste-efectividad del tratamiento con PTN, así como identificar las marcas comercializadas actualmente en España. 


\section{ORIGEN Y FUNDAMENTO DEL TRATAMIENTO CON PRESIÓN TÓPICA NEGATIVA}

Las heridas crónicas son aquellas que no evolucionan más allá de la fase inflamatoria debido a un desequilibrio entre los factores de crecimiento y las proteasas. Pueden definirse como cualquier solución de continuidad en la superficie corporal que no cicatriza a la velocidad esperada ni con el tratamiento convencional, o que recurre una vez cicatrizada (3). Esto puede ser debido a múltiples factores, como la presencia exagerada de citocinas proinflamatorias, la disminución de los factores de crecimiento, la alteración en el depósito de colágeno y de la matriz, la alteración de la proliferación celular y de la síntesis proteica, y el aumento de la apoptosis. La causa por la cual una herida se transforma en crónica es multifactorial. Cuando la presión de oxígeno se halla por debajo de los $40 \mathrm{mmHg}$, disminuye y se enlentece la producción de colágeno.

El tratamiento con PTN en su aplicación clínica apareció en 1940, aunque ha sido en la última década cuando su popularidad ha aumentado. Tal como describieron en 1997 Argenta y Morykwas (4), consiste en la aplicación en el lecho de la herida de una esponja porosa conectada a través de un tubo a una bomba de vacío y cubierta con un apósito adherente. Se consigue de esta forma aplicar una presión controlada continua o intermitente, en un intervalo de valores de entre $50 \mathrm{y}$ $125 \mathrm{mmHg}$ sobre el lecho de la herida. Esa espuma es un elemento crucial en el proceso y condujo a Kinetic Concepts Inc $\left(\mathrm{KCI}^{\odot}\right)$ a desarrollar un sistema comercial por el que el tratamiento con PTN es más conocido a nivel mundial: el sistema de cierre al vacío (V.A.C. $\left.{ }^{\circledR}\right)$. Este sistema es el empleado por la mayoría de marcas comerciales para la aplicación tópica de la presión negativa, puesto que el mecanismo para lograrlo es precisamente hacer un vacío, de ahí que el término más común para este tipo de terapias sea "sistema de vacío".

A causa de la presión, el volumen de la espuma se reduce, lo que da lugar a la extensión de las células, la contracción de la herida y la eliminación del líquido. La presión subatmosférica, que también se conoce como presión negativa, es una denominación que se emplea para describir una presión inferior a la presión atmosférica normal. Ésta puede conseguirse extrayendo moléculas de gas fuera de la zona de interés (como la zona de una herida), por ejemplo con un sistema de aspiración. La base de esta técnica está en hacer progresar la herida crónica de la fase inflamatoria a la fase proliferativa.

Existen diversos mecanismos que podrían ser los responsables de los efectos beneficiosos del tratamiento (5):

- Aumento del flujo sanguíneo local.

- Reducción del edema.

- Estimulación de la formación de tejido de granulación.

- Estimulación de la proliferación celular.

- Eliminación de inhibidores solubles de la cicatrización de la herida.

- Reducción de la carga bacteriana.

- Acercamiento de los bordes de la herida entre sí.

Las fases por las que pasa la herida son: contracción de la herida (macrodeformación), estabilización del ambiente de la herida, disminución del edema y exudado, y microdeformación.

\section{$\checkmark$ INDICACIONES}

Para el uso del tratamiento con PTN en una herida hay que aplicar los mismos criterios que se aplicarían para la selección de cualquier intervención: debe elegirse si constituye el método más coste-efectivo y más eficaz desde el punto de vista clínico. Los resultados del tratamiento deben reevaluarse adecuadamente.

Está indicado en heridas, lesiones y dehiscencias quirúrgicas de grandes dimensiones, elevada complejidad o con un retraso considerable en la cicatrización, como serían las úlceras varicosas, diabéticas o cualquier lesión con pérdida de continuidad (6). También se indica en heridas que están en relación con síndromes compartimentales, tanto en el abdomen como en las extremidades (7). Se indica con restricciones en pacientes con cáncer, con terapia anticoagulante, con sangrado activo y en los que presentan una fístula enterocutánea. Está contraindicado su uso en pacientes con osteomielitis, con presencia de tejido necrótico o con trastornos psiquiátricos $(8,9)$. No deben colocarse las esponjas sobre arterias o venas por el alto riesgo de que se produzca un sangrado masivo y de difícil control (10).

En países como EE.UU., Reino Unido y Alemania es el tratamiento de primera elección para UPP, lesiones de origen vascular o pie diabético, sin que tengan que llegar a ser lesiones de larga evolución, tanto en el medio hospitalario como en Atención Primaria. En España su indicación es hospitalaria, de prescripción médica y en lesiones complejas, fundamentalmente dehiscencias con importante pérdida de continuidad y de grandes dimensiones.

\section{COMPONENTES DEL SISTEMA}

El sistema en general está compuesto por un apósito (la mayoría de espuma de poliuretano, que suele ser de color negro, o alcohol polivinílico, que suele ser de color blanco; pueden llevar plata) o gasa. A este apósito se conecta, mediante un tubo flexible y una ventosa, un sistema de vacío o bomba que será el encargado de generar el vacío. El apósito queda fijado a la piel gracias a una película de poliuretano transparente que a su vez sella el sistema de vacío. La bomba de vacío lleva incorporado un reservorio en el que se almacenará el exudado extraído durante la succión, que puede ser continua o intermitente. De manera muy burda podría decirse que el sistema es similar al sellado al vacío de los alimentos, sólo que en este caso además se extrae el exudado.

En la figura 1 se explica cómo es el procedimiento de colocación del apósito (tomado de www.kci-medical.com, con autorización).

\section{y COSTE-EFECTIVIDAD}

La mayoría de los estudios han sido realizados empleando sistemas de vacío como terapia con PTN, por lo que en este apartado hablaremos

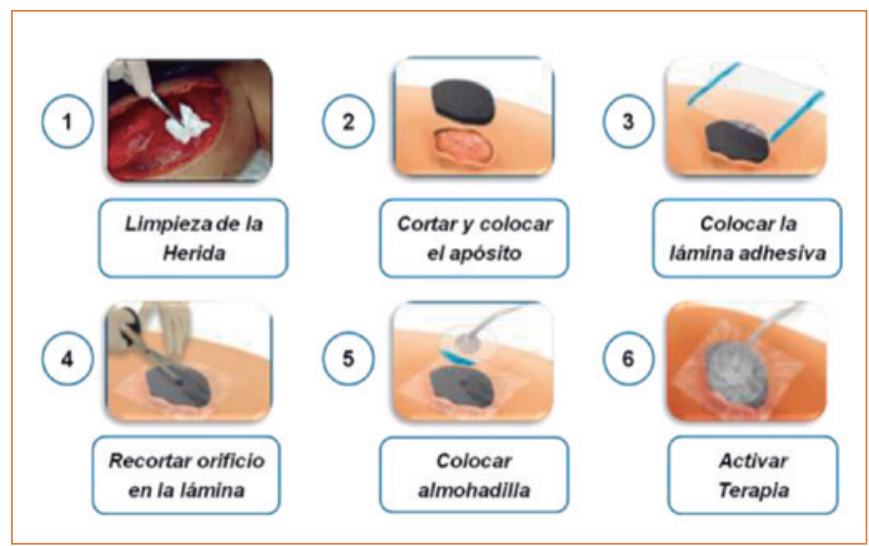

Figura 1. Pasos para la colocación del apósito del sistema V.A.C. ${ }^{\circledR}$ (reproducido con autorización). 
exclusivamente de ellos. Este sistema se considera caro en relación con otras medidas terapéuticas como los apósitos, de ahí que su uso sea restringido, especialmente fuera del ámbito hospitalario (11). Sin embargo, los costes del apósito en general representan un porcentaje pequeńo del coste total del tratamiento de las heridas crónicas (12), puesto que el mayor coste se deriva del tiempo de enfermería, de la hospitalización y del abordaje de las complicaciones. A la hora de estudiar el coste-efectividad de los apósitos, hay una tendencia a centrarse en el coste de los apósitos más que en el coste del tratamiento, en el cual pueden influir otros factores como el tiempo transcurrido hasta la cicatrización. Centrarse sólo en lo que cuesta un apósito en vez de hacer estudios sobre lo que cuesta un tratamiento completo (por desgracia el tiempo de dedicación de enfermería se valora en pocos estudios cuando posiblemente sea un factor coste-efectividad decisivo) es fruto posiblemente de una cultura de gestión cortoplacista y sesgada, cuando la lógica indica que se deberían hacer estudios integrales: no valorar sólo lo que cuesta un apósito, sino valorar cuánto cuesta el proceso de cicatrización completo. Parece que existen evidencias de que la PTN reduce el tiempo de hospitalización, los procedimientos quirúrgicos para implantar colgajos e injertos, los desbridamientos repetitivos y los reingresos. De hecho, hay estudios que corroboran un ahorro en los costes de incluso 3100 dólares (13) en hospitalización por heridas traumáticas y de 891,9 en las crónicas (14).

A la hora de analizar otros tratamientos frente a la cura convencional existen estudios que apuestan por la implementación del coste-efectividad. En un informe detallado de la European Wound Management Association (EWMA) de 2003, Franks y Posnett analizaron el coste-efectividad del tratamiento de compresión para las úlceras venosas en extremidad inferior (12). Basándose en los costes estimados del tratamiento semanal, el coste total del tratamiento a lo largo del tiempo fue menor cuando se utilizó el tratamiento más caro (es decir, la compresión) que cuando se utilizó un apósito convencional más barato (1697 euros frente a 3558 euros por úlcera cicatrizada). La principal causa de la diferencia fue que el tiempo transcurrido hasta la cicatrización y el número total de cambios de apósitos fueron menores con el tratamiento de compresión.

Un análisis realizado por Harding y cols. también indicó que los apósitos representan entre el $4 \%$ y el $29 \%$ del coste total del tratamiento de las UPP (15). Los resultados de ensayos aleatorizados controlados en los que se comparó el tratamiento con PTN o sistemas de vacío con otros apósitos en pacientes con úlceras de pie diabético parecen corroborar la teoría de que "lo más barato a veces sale más caro" (16-19).

Un estudio prospectivo y comparativo llevado a cabo con el tratamiento con PTN en UPP en el que se han comunicado las tasas de cicatrización fue realizado por Ford y cols. (20). Los autores concluyeron, aunque con cautela, que el tratamiento con PTN fue más eficaz, con una reducción en el tiempo de cicatrización, que otros tratamientos convencionales basados en sistema de curas en ambiente húmedo. En otro estudio se revisó la bibliografía sobre la eficacia del tratamiento de las UPP con PTN, con alginatos y con hidrocoloides (21). Los resultados mostraron que el 93\% de las heridas tratadas con PTN cicatrizó, frente al 63\% de las heridas tratadas con hidrocoloides de manera estadísticamente significativa. Philbeck y cols. calcularon el coste del tratamiento de las UPP utilizando estimaciones de las reducciones diarias del área de las heridas con el tratamiento con PTN y con el tratamiento convencional (22). Los costes del tratamiento hasta la cicatrización fueron de 23465 dólares (18 155 euros) con el tratamiento convencional y de 14546 dólares (11 256 euros) con el tratamiento con PTN. A pesar de ello, es un estudio que debe tomarse con cautela por algunos sesgos metodológicos.
El sistema más estudiado y extendido es la terapia V.A.C. ${ }^{\circledR}$, de la que existen más de 600 artículos publicados y revisados por expertos, incluidos los datos de economía sanitaria, y más de 22 estudios de nivel 1 RCT (estudios controlados aleatorizados) (23). El informe ECRI (29 de junio de 2000) indicaba que: "Los resultados de los pocos estudios que comparan el V.A.C. ${ }^{\circledR}$ con tratamiento tradicional de cura húmeda sugieren que la terapia promueve una cicatrización más rápida, que se traduce en ahorro de costes, particularmente en el tratamiento de heridas crónicas" (23). Según la compañía $\mathrm{KCI}^{\odot}$, la duración habitual del tratamiento de una herida compleja con el sistema V.A.C. ${ }^{\circledR}$ se estima en 21 días (tiempo medio estimado para su cicatrización completa o casi completa), lo que en comparativa con un tratamiento convencional que puede alargarse meses supondría para un hospital de tamaño medio (unos 7 días de terapia por año y por cama hospitalaria) un ahorro entre $900000 €$ y $2200000 €$ al año, estimando tanto el coste del tratamiento convencional como el tiempo de enfermería, la reducción en hospitalización y el abordaje de las complicaciones (23).

No obstante, aunque todos los estudios apuntan claramente a los beneficios multifactoriales del tratamiento con PTN (reducción de tiempos de cicatrización, mejora en la calidad de vida, reducción de complicaciones, reducción de costes, etc.) según un metaanálisis reciente de Cochrane, sólo dos estudios que cubrían los requerimientos metodológicos indicaron que el tratamiento con PTN reduce de manera efectiva el tamańo de las heridas, aunque esta conclusión tiene poca relevancia estadística. Los estudios realizados hasta la fecha incluyen poblaciones muy reducidas y presentan limitaciones metodológicas importantes, por lo que aún debe seguir investigándose en esta línea para poder hablar de manera fiable de coste-efectividad (24).

\section{$\searrow$ MARCAS COMERCIALES EN ESPAÑA}

La compañía $\mathrm{KCI}^{\odot}$ comercializa a nivel mundial la TPN desde hace más de 35 años, pero en España desde hace unos 12. Es la marca más extendida y es conocida en nuestros hospitales como el sistema V.A.C. ${ }^{\circledR}$ Ofrece una amplia gama de soluciones y respuestas, con espumas de poliuretano, alcohol polivinílico, asociaciones con plata, abordaje para el abdomen abierto, para fístulas, con diversidad de bombas de vacío y de contenedores. En la figura 2 se muestra un dispositivo y algunos elementos que componen el sistema V.A.C. ${ }^{\circledR}$ Therapy.

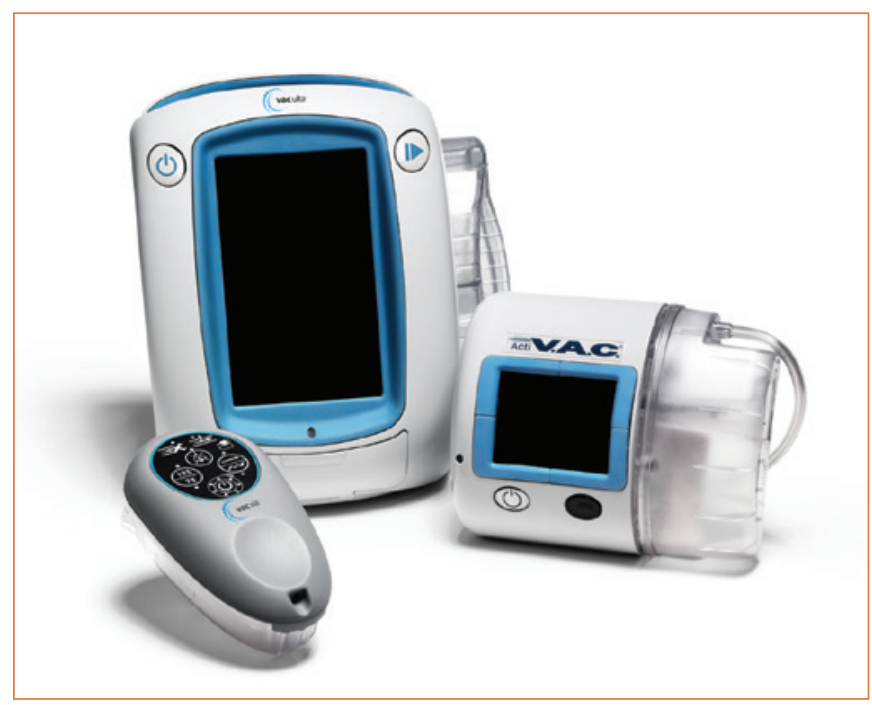

Figura 2. Sistema V.A.C. ${ }^{\circledR}$ Therapy (reproducido con autorización). 


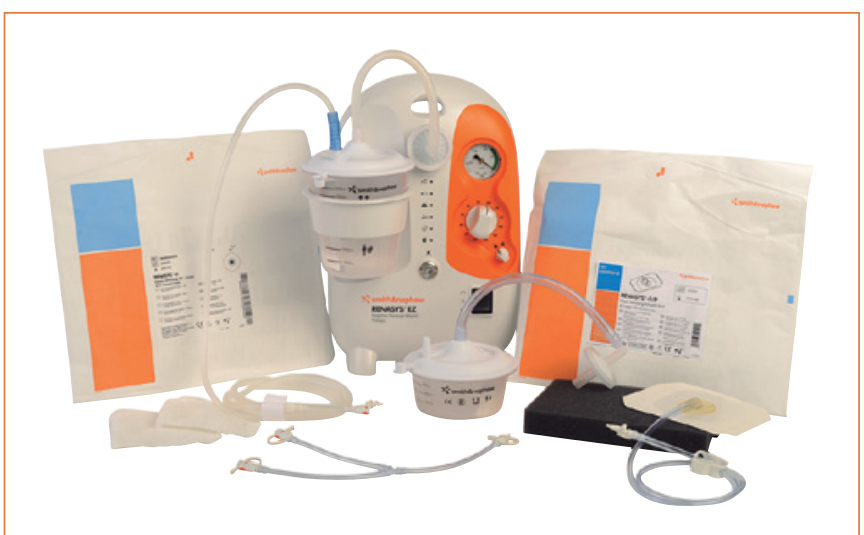

Figura 3. Sistema Renasys ${ }^{\circledR}$ de Smith\&Nephew ${ }^{\odot}$ (reproducido con autorización).

Otro sistema de posterior comercialización en nuestro país es el sistema de presión negativa Renasys ${ }^{\circledast}\left(\right.$ Smith\&Nephew $\left.{ }^{\odot}\right)$, que emplea apósitos de espuma de poliuretano y gasa, y dispone también de un sistema portátil para lesiones menores o ambulatorias $\left(\mathrm{PICO}^{\circledR}\right)$ que está teniendo buena acogida, sobre todo en Atención Primaria. En la figura 3 se muestra la bomba de vacío del sistema Renasys ${ }^{\oplus}$. En breve también se comercializará el sistema $V_{y v a n o}{ }^{\oplus}$ de Hart-

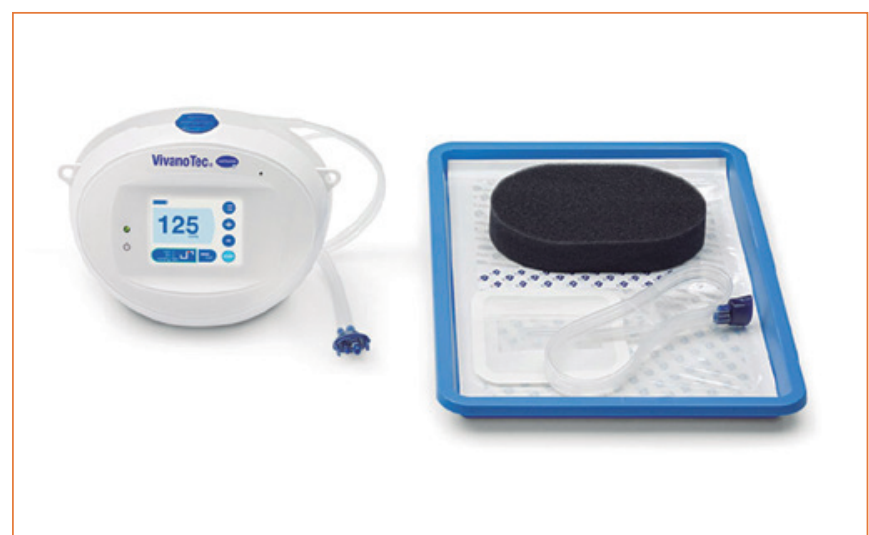

Figura 4. Sistema Vyvano ${ }^{\circledR}$ de Hartmann ${ }^{\odot}$ (reproducido con autorización).

mann ${ }^{\odot}$, con una espuma de poliuretano, de reciente lanzamiento en Europa, y un sistema muy sencillo de aplicación. En la figura 4 se muestra una imagen de este sistema.

Y por último, y con buena acogida gracias al uso de la tecnología $S_{\text {Safetac }}{ }^{\oplus}$ con buenos resultados en la protección de la piel perilesional, el sistema Avance ${ }^{\oplus}$ de Mölnlycke Health Care ${ }^{\odot}$, que emplea como película transparente para el sellado una lámina de silicona

\section{У BIBLIOGRAFÍA}

1. Soldevilla JJ, Torra i Bou JE, Verdú J, Martínez Cuervo F, López Casanova P, Rueda J y cols. 2. ${ }^{\circ}$ Estudio Nacional de Prevalencia de Úlceras por Presión en España, 2005. Epidemiología y variables definitorias de las lesiones y pacientes. Disponible en: www.gerokomos.com

2. Banwell PE. Topical negative pressure therapy in wound care. J Wound Care 1999: 8: 79-84.

3. Wysocki AB. Wound fluids and the pathogenesis of chronic wounds. J Wound Ostomy Continence Nurs 1996; 23: 283-90.

4. Argenta LC, Morykwas MJ. Vacuum-assisted closure: a new method for wound control and treatment: clinical experience. Ann Plast Surg 1997; 38: 56376.

5. Philbeck TE Jr, Whittington KT, Millsap MH, Briones RB, Wight DG, Schroeder WJ. The clinical and cost effectiveness of externally applied negative pressure wound therapy in the treatment of wounds in home healthcare Medicare patients. Ostomy Wound Manage 1999; 45: 41-50.

6. Timmers MS, Le Cessie S, Banwell P, Jukema GN. The effects of varying degrees of pressure delivered by negative-pressure wound therapy on skin perfusion. Ann Plast Surg 2005; 55: 66571; discussion 1097-98

7. Morykwas MJ, Faler BJ, Pearce DJ, Argenta LC. Effects of varving levels of subatmospheric pressure on the rate of granulation tissue formation in experimental wounds in swine. Ann Plast Surg 2001; 47: 547-51.

8. Saxena V, Hwang CW, Huang S, Eichbaum O, Ingber D, Orgill DP. Vacuum-assisted closure: microdeformations of wounds and cell proliferation. Plast Reconstr Surg 2004; 114: 1086-96.
9. Greene AK, Puder M, Roy R, Arsenault D, Kwei S, Moses MA, Orgill DP. Microdeformational wound therapy: effects on angiogenesis and matrix metalloproteinases in chronic wounds of 3 debilitated patients. Ann Plast Surg 2006; 56: 418-22.

10. Stechmiller JK, Kilapadi DV, Childress B, Schultz GS. Effect of vacuum-assisted closure therapy on the expression of cytokines and proteases in wound fluid of adults with pressure ulcers (letter to editor). Wound Rep Regen 2006; 14: 371-4.

11. Newton H, Benbow M, Hampton S, Beldon P, Butcher M, Baxter $H$. TNP therapy in the community: findings of a national survey. Wounds UK 2006; 2: 31-5.

12. Franks PJ, Posnett J. Cost-effectiveness of compression therapy. En: European Wound Management Association (EWMA). Position Document: Understanding compression therapy. London: MEP Ltd, 2003, pp. 8-10.

13. Kaplan M. Negative Pressure Fundamental Principles. 2. ${ }^{\circ}$ Encuentro World Union of Wound Healing Societies. París, 2004. Wounds 2004; 16 (12A Suppl): 4S.

14. Philbeck TE, Whittington KT, Millsap MH, Briones RB, Wight DG, Schroeder WJ. The clinical and cost effectiveness of externally applied negative pressure wound therapy in the treatment of wounds in home healthcare Medicare patients. Ostomy Wound Manage 1999; 45: 41-50.

15. Harding K, Cutting K, Price P. The cost-effectiveness of wound management protocols of care Br J Nurs 2000: 9 (19 Suppl): S6-S24.

16. McCallon SK, Knight CA, Valiulus JP, Cunningham MW, McCulloch JM, Farinas LP. Vacuum-assisted closure versus sali- ne-moistened gauze in the healing of postoperative diabetic foo wounds. Ostomy Wound Manage 2000; 46: 28-32, 34

17. Eginton MT, Brown KR, Seabrook GR, Towne JB, Cambria RA. A prospective randomized evaluation of negative-pressure wound dressings for diabetic foot wounds. Ann Vasc Surg 2003; 17: 645 9. Epub 2003: Oct 13

18. Armstrong DG, Lavery LA; Diabetic Foot Study Consortium. Negative pressure wound therapy after partial diabetic foot amputation: a multicentre, randomized controlled trial. Lancet 2005: 366: 1704-10.

19. Joseph E, Hamori CA, Bergman S, Roaf C, Swan N. A prospective randomized trial of vacuum-assisted closure versus standard therapy of chronic non-healingwounds. Wounds 2000; 12:60-7.

20. Ford CN, Reinhard ER, Yeh D, Syrek D, De Las Morenas A, Bergman SB y cols. Interim analysis of a prospective, randomized trial of vacuum-assisted closure versus the Healthpoint system in the management of pressure ulcers. Ann Plast Surg 2002; 49: 55-61.

21. Smith $\mathrm{N}$. The benefits of VAC therapy in the management of pressure ulcers. Br J Nurs 2004; 13: 1359-65.

22. Philbeck TE Jr, Whittington KT, Millsap MH, Briones RB, Wight DG, Schroeder WJ. The clinical and cost effectiveness of externally applied negative pressure wound therapy in the treatment of wounds in home healthcare Medicare patients. Ostomy Wound Manage 1999; 45: 41-50.

23. Página web de K.C. ${ }^{\odot}$ [acceso 15 de diciembre de 2012]. Disponible en: www.kci-medical.com.

24. Evans $D$, Land $L$. Topical negative pressure for treating chronic wounds. Cochrane Database Syst Rev 2001;CD001898. 